\title{
DESEMPENHO NO FUTEBOL: ESTIMULAÇÃO CEREBRAL E NEURÔNIOS ESPELHO
}

\author{
Luiza Pinheiro Anhanha Branco', Gabriel dos Santos Guilherme', Ricardo Costa Abrantes \\ Junior ${ }^{1}$, Vernon Furtado da Silva ${ }^{2}$ \& Mauricio Rocha Calomeni ${ }^{2 *}$
}

BRANCO, L.P.A.; GUILHERME, G.S.; ABRANTES JUNIOR, R.C.; SILVA, V.F. \& CALOMENI, M.R. Desempenho no futebol: estimulação cerebral e neurônios espelho. Perspectivas Online: Biológicas \& Saúde, v.10, n.34, p.16-32, 2020.

\section{RESUMO}

O objetivo foi comparar os efeitos da associação do treinamento mental, ativação dos neurônios espelho e estimulação cerebral não invasiva sobre o desempenho técnico específico do futebol. 17 atletas da equipe de futebol feminino profissional do Flamengo, disputando o campeonato brasileiro de futebol de 2019, de 19 a 34 anos, foram divididas randomicamente em dois grupos, grupo Neurônios Espelhos (NE) (n=9) e Estimulação Cerebral (EC) $(\mathrm{n}=8)$. Foram avaliadas a atividade cerebral, a velocidade de processamento mental, e a habilidade motora em uma tarefa específica. Ambos os grupos mantiveram a rotina de treino prevista pela comissão técnica. Além disto, o NE recebeu um arquivo de vídeo com imagens do desempenho de uma tarefa motora técnica, editado com um áudio do protocolo de treinamento mental, já o EC recebeu arquivo de áudio com o protocolo de estimulação cerebral por batidas binaurais mixado com os comandos para o treinamento mental. As atletas foram orientadas a executarem os arquivos recebidos sempre 15 minutos antes dos treinamentos convencionais durante 7 semanas. Comparações dentro dos grupos mostraram que NE melhorou significativamente o tempo de execução da tarefa $(p=0,01)$. Contudo em todas as outras comparações não foram observadas diferenças estatisticamente significativas tanto dentro quanto entre os grupos $(p>0,05)$. Acredita-se que a forma selecionada para efetivação das intervenções possa ter impactado negativamente para resultados mais significativos. Contudo, o treinamento mental associado a ativação dos neurônios espelhos se mostrou mais eficiente para redução do tempo de execução da tarefa técnica e aumento na precisão ao acertar o alvo pré-determinado.

Palavras-chave: Neurônios Espelhos; Futebol; Estimulação Cerebral.

\footnotetext{
${ }^{1}$ Institutos Superiores de Ensino do CENSA - ISECENSA - Laboratório de Biociências da Hotricidade Humana - LABIMH Rua Salvador Correa, 139, Centro, Campos dos Goytacazes, RJ, CEP: 28035-310, Brasil;

${ }^{2}$ Universidade Federal de Rondônia - UNIR - Departamento de Educação Física - Av. Presidente Dutra, 2965 - Centro CEP: 76801-974 - Porto Velho - RO.

$\left(^{*}\right)$ e-mail: mauriciocalomeni@,isecensa.edu.br
} 


\title{
FOOTBALL PERFORMANCE: BRAIN STIMULATION AND MIRROR NEURONS
}

\author{
Luiza Pinheiro Anhanha Branco', Gabriel dos Santos Guilherme ${ }^{1}$, Ricardo Costa Abrantes
} Junior ${ }^{1}$, Vernon Furtado da Silva ${ }^{2}$ \& Mauricio Rocha Calomeni ${ }^{2 *}$

BRANCO, L.P.A.; GUILHERME, G.S.; ABRANTES JUNIOR, R.C.; SILVA, V.F. \& CALOMENI, M.R. Football performance: brain stimulation and mirror neurons. Perspectivas Online: Biológicas \& Saúde, v.10, n.34, p.16-32, 2020.

ABSTRACT

\begin{abstract}
The goal's study was to compare the effects of the association of mental training, mirror neuron activation, and non-invasive brain stimulation in the specific technical performance of soccer. 17 female professional athletes of Flamengo that were playing the Brazilian Soccer Championship of 2019, with an age range 19 to 34 years, were randomly divided into two groups, Mirror Neurons group (NE) $(\mathrm{n}=9)$ and Brain Stimulation group (EC) $(n=8)$. They had evaluated the brain activity, the mental processing speed, and the motor ability in a specific task. Both groups kept the planned training by the technical committee. Besides that, the NE received a video file with images of the motor performance in the task edited with the mental training sound, already the EC group received a sound file with the brain
\end{abstract}

stimulation protocol by binaural beats mixed with mental training commands. Was determined to athletes executed the files always 15 minutes before conventional training by 7 weeks. Comparisons into the groups showed that NE improved significantly the execution time of the task $(p=0,01)$. However, in all other comparisons, no statistically significant differences were observed both within and between groups $(\mathrm{p}>0,05)$. We believed that the selected way for the athletes got the interventions may have had a negative impact to produce more positive results. However, the mental training associated with mirror neuron activation showed more efficiency in the reduction of the task time execution and an increase in accuracy when hitting the predetermined target.

Keywords: Mirror Neurons; Soccer; Brain Stimulation.

\footnotetext{
${ }^{1}$ Institutos Superiores de Ensino do CENSA - ISECENSA - Laboratório de Biociências da Hotricidade Humana - LABIMH Rua Salvador Correa, 139, Centro, Campos dos Goytacazes, RJ, CEP: 28035-310, Brasil;

${ }^{2}$ Universidade Federal de Rondônia - UNIR - Departamento de Educação Física - Av. Presidente Dutra, 2965 - Centro CEP: 76801-974 - Porto Velho - RO.

$\left.{ }^{*}\right)$ e-mail: mauriciocalomeni@isecensa.edu.br
} 


\section{INTRODUÇÃO}

O desempenho no desporto, principalmente no desporto coletivo como o futebol profissional, é determinado pela associação dos aspectos físicos, motores, cognitivos e psicológicos, de modo que para o desenvolvimento satisfatório desses fatores se faz necessário além da aptidão cardiorrespiratória, a aptidão neuromuscular, a tomada de decisão, a técnica dos fundamentos do jogo, bem como a capacidade mental de cada atleta (FORTES et al, 2017).

Especificamente no futebol profissional o desempenho técnico é um dos fatores considerado como fundamental para o alcance de resultados positivos, levando treinadores a desenvolver métodos de treinamento de habilidades específicas que são praticadas exaustivamente em várias circunstâncias ou situações de jogo. Sendo assim, apesar do grande foco que diferentes técnicos e clubes dão ao treinamento de elementos motores, pesquisas na área da neurociência cognitiva revelam a relevância de funções cognitivas no controle do movimento voluntário (CONDE et. al., 2012).

De fato o aprimoramento das capacidades motoras voluntárias através da estimulação de funções cognitivas e mentais tem sido verificado (RIBEIRO JUNIOR et al, 2010; FORTES et al, 2017). O que tem produzido cada vez mais consenso no meio acadêmico e profissional de que a perícia esportiva, bem como o seu rendimento, é influenciada pela associação dos componentes cognitivos e motores (MATIAS e GRECO, 2010). E, dentre os componentes cognitivos mais relevantes para o esporte está à tomada de decisão, pois implica escolher a solução adequada em situações complexas, ambíguas e urgentes (RIPOLL, 2011). Sendo assim, Tenenbaum (2003) aborda a tomada de decisão sobre uma perspectiva de processamento de informação, pois comportamentos motores em situações competitivas é resultado da codificação de pistas ambientais relevantes e pertinentes, captadas através de estratégias de atenção e interação entre as memórias de trabalho e longo prazo.

Dentre os métodos e estratégias com objetivo de auxilio e aprimoramento das capacidades mentais e psicomotoras de quaisquer indivíduos destacam-se especificamente para este trabalho, a ativação do sistema de neurônios espelho (NE), o treinamento mental (TM) e a estimulação cerebral não invasiva (EC) sob a perspectiva do desempenho desportivo. Neste cenário, o sistema de neurônios espelho chama atenção por ser o responsável pela ponte intercerebral de comunicação e conexões em múltiplos níveis do cérebro que permitem ao indivíduo imitar através da observação e percepção (LAMEIRA e PEREIRA JR, 2006; RIZZOLATTI, FOGASSI e GALLESE, 2006 apud. FERNANDES, 2010). Isso contribui de forma significativa para o processo de aprendizagem uma vez que permite ao indivíduo identificar características importantes de uma tarefa a ser executada e extrair informações acerca de um padrão de coordenação motora exigido nesta tarefa (SANTOS-NAVES et. al. 2014).

Quanto à prática mental (TM), o treinamento em suma tem buscado possíveis melhoras nas habilidades psicológicas, técnicas e até mesmo motoras, por compreender a mentalização e racionalização intensiva de uma sequência de movimentos a serem executados, com o objetivo de aperfeiçoamento do seu processamento (WEINECK, 1999 apud OLIVEIRA, 2007). O treinamento mental tem sido proposto como meio de contribuir para aprendizagem de habilidades motoras, bem como para melhoria do desempenho e aperfeiçoamento de habilidades motoras no esporte em geral (PEREIRA, SANTOS e CILLO, 2018) e no futebol (FILGUEIRAS et al, 2012). 
Dentre os instrumentos de estimulação cerebral conhecidos, a conjugação da estimulação por luz e batida binaural tem se mostrado eficiente em investigações que analisaram seus efeitos na melhoria funções motoras deterioradas por AVC (CALOMENI et al, 2013), na ativação dos neurônios do lobo temporal de autistas (SILVA et al, 2016), na melhoria da concentração de escolares (LIMA et al, 2016), na melhora da memória de trabalho de atenção concentrada de criança hiperativa (CALOMENI et al, 2008; LIMA et al, 2014), e, no desenvolvimento cinestésico de crianças (LIMA e CARDOSO, 2017). Além disso, já foi proposta como auxiliar na maximização do próprio treinamento mental, por ter a capacidade de potencializar a atividade cerebral (AREAAS NETO, 2010). Sendo que o efeito de arrastamento das ondas cerebrais produzido pela utilização das batidas binaurais de forma isolada também já foi verificado (CALOMENI et al, 2017).

Diante disto o objeto desse estudo foi verificar como a associação do treinamento mental com a ativação dos neurônios espelho e estimulação cerebral não invasiva puderam influenciar no desempenho de uma técnica esportiva específica, e assim, caso alguma hipótese se confirme oferecer aos profissionais de educação física, técnicos e treinadores na área do futebol ou não, respaldo para a realização de trabalhos relacionados direta e indiretamente às estratégias pautadas no treinamento mental, estimulação cerebral e sistema de neurônios espelho (SNE) com o objetivo de promover a melhoria do desempenho técnico dos seus atletas.

\section{METODOLOGIA}

Delineamento experimental verdadeiro consiste em qualquer delineamento usado em pesquisas experimentais em que os grupos são formados randomicamente e há controle da maioria das fontes de invalidação (THOMAS, NELSON e SILVERMAN, 2009). A presente pesquisa se enquadra nesse delineamento uma vez que foram formados randomicamente dois grupos que passaram por intervenções distintas. A pesquisa foi avaliada e aprovada pelo comitê de ética em pesquisa com seres humanos dos Institutos Superiores de Ensino do CENSA, com o parecer consubstanciado $\mathrm{n}^{\mathrm{o}} 2.561 .404$.

\subsection{Amostra}

A amostra total foi composta pelo universo de atletas de futebol profissional $(n=22)$ mantidas pelo clube de Regatas Flamengo em parceria com a Marinha do Brasil que fornecia toda estrutura de treinamento existente no Centro de Educação Física Almirante Adalberto Nunes (CEFAN), todas do gênero feminino, com faixa etária de 19 a 34 anos de idade, em período de competição no Campeonato Brasileiro de 2019. Desse total, a amostra final foi composta por 17 atletas que atenderam aos critérios de inclusão e exclusão determinados. Foram amplamente esclarecidas sobre os objetivos e procedimentos do estudo de forma que concordaram voluntariamente em participar do estudo através da assinatura do termo de consentimento livre e esclarecido aprovado previamente pelo comitê de ética em pesquisa com seres humanos.

\subsubsection{Critérios de Inclusão}

Foram incluídas jogadoras com no mínimo 5 anos de prática no esporte, regularmente registradas na respectiva federação esportiva, e que estivessem frequentando regularmente as sessões de treinamento. Vale ressaltar que todas as atletas atenderam a esses critérios. 


\subsubsection{Critérios de exclusão}

Foram excluídas atletas lesionadas durante o período do estudo, que sofressem de transtornos auditivos não corrigidos por equipamento externo e que não concordassem voluntariamente com a participação no estudo. Sendo assim, 4 atletas foram excluídas por estarem, no momento da avaliação inicial, sendo atendidas no departamento médico devido a alguma lesão.

\subsection{Instrumentos}

Para avaliação da atividade cerebral foi utilizado um equipamento de eletroencefalograma produzido pela Neurotec, modelo Neuromap EQSA260, produzido no Brasil e destinado a registrar em tempo real, frequências de ondas cerebrais em pontos específicos do córtex cerebral, relacionados com funções e processamento sensório-motor, atenção e processamento mental. Os pontos de interesse do estudo foram determinados de acordo com o sistema internacional 10-20, indicado pela Sociedade Brasileira de Neurofisiologia Clínica (SILVA et. al., 2016), e, as faixas de frequência registradas foram as ondas Alfa e Beta1/SMR que abrange as frequências de 8 a $15 \mathrm{~Hz}$.

Sendo assim foram selecionados os pontos CZ, C3 e C4 (córtex motor esquerdo, direito e central). Além destes foram utilizados os pontos A1 e A2 como referência para os demais, e, mais dois pontos como terra. Os pontos $\mathrm{C} 3, \mathrm{C} 4 \mathrm{e} \mathrm{Cz}$ foram escolhidos por representar o córtex motor que está funcionalmente relacionado à preparação e execução motora (DARONCH, 2016). Instrumento semelhante tem sido utilizado em outras pesquisas na área da neurociência (CALOMENI et al, 2013; CALOMENI et. al., 2017). Ainda em relação à efetividade deste instrumento, a fim de se evitar interferências e artefatos no sinal, antes do início do exame foi feita a limpeza de cada ponto no couro cabeludo com um algodão embebido com álcool $70 \%$, solicitado à avaliada que mantivessem braços e pernas descruzados durante a realização do exame, e utilização de um tapete de material isolante (borracha) posicionado no solo abaixo dos pés das atletas para que as mesmas não tocassem o solo durante o exame. Além disso, cada exame só era iniciado quando se a taxa de impedância estivesse abaixo de $20(\mathrm{~K} \Omega)$.

Para se mensurar a velocidade de tomada de decisão das atletas, foi utilizado um software desenvolvido para registrar o tempo despendido entre a apresentação de um estímulo na tela do computador e a resposta motora apropriada. O referido teste, disponibilizado gratuitamente na internet, foi desenvolvido por Okazaki (2011) para ser aplicado através de um notebook, sendo possível configurar o tipo de estímulo (letras, números, símbolos ou cores), além das possibilidades de respostas. Isso permite que se ajuste o nível de dificuldade do teste. A velocidade de tomada de decisão no contexto esportivo, como preditivo para velocidade de processamento de informação, já foi inferida através do tempo de reação motora (BISCAIA et al, 2018), pois depende da percepção e interpretação do estímulo para que seja programada e executada a resposta apropriada (GONÇALVES, 2014).

O protocolo de estimulação cerebral não invasiva por batidas binaurais teve como objetivo o treinamento das ondas cerebrais Alfa e Beta/SMR, e consistiu na utilização de um aparelho eletrônico conhecido como sintetizador de ondas corticais, denominado Sirius e fabricado pela Mind Place. A estimulação cerebral por batidas binaurais consiste na emissão de dois sons chamados de batidas binaurais, que emitem frequências parecidas, sendo assim transportado de forma neurológica à formação reticular, que é capaz de controlar e regular o nível da consciência, atenção e estresse, que são fatores importantes para uma boa 
aprendizagem (CALOMENI et. al., 2018). O referido equipamento possui uma sessão de estimulação cerebral pré-programada específica para induzir estados de concentração com duração de 15 min. A referida sessão foi convertida em arquivo MP3 de forma que pudesse ser executada simultaneamente com o protocolo de treinamento mental.

O protocolo de treinamento mental foi desenvolvimento especificamente para esse estudo de forma a promover nas atletas a "mentalização e racionalização intensiva da sequência dos movimentos a serem executados, a fim de aperfeiçoar o seu processamento" (WEINECK, 1999 apud. OLIVEIRA, 2007). Ou seja, foi gravada uma série de comandos com o objetivo de fazer com que as atletas revivessem mentalmente todos os detalhes da tarefa motora utilizada para verificação da habilidade motora. Optou-se pela gravação dos comandos do protocolo para que não houvesse variações na forma como os comandos para o treinamento mental fossem passados para as atletas. Um texto contendo uma voz de comando com estímulos para mentalização foi gravado de forma que, em intervalos de 2 a 3 minutos um comando para mentalização de um aspecto específico da tarefa fosse estimulado. A gravação do protocolo de treinamento mental teve um tempo total de 15 minutos.

Para a avaliação da habilidade motora, uma tarefa específica deveria ser realizada por cada atleta. Para isso uma tarefa foi estruturada especificamente para essa pesquisa a partir de propostas dadas pelo técnico e comissão técnica com o objetivo de se determinar o nível ideal de complexidade a partir das limitações técnicas da equipe apontadas pelos mesmos. Sendo assim, foi determinada uma tarefa que realmente representasse um desavio em termos técnicos para as atletas. Dessa forma, partindo de um ponto pré-determinado por um cone, localizado a 9 metros e quinze centímetros da linha do meio do campo, a atleta deveria conduzir a bola em direção ao gol, fazendo movimento de passar por entre 4 balizas (movimento de zigue-zague), onde a primeira baliza estava localizada a 10 metros do ponto de partida e cada baliza posicionada a 1 metro de distância uma das outras. No fim destes movimentos, a atleta deveria realizar um deslocamento lateral em direção ao centro do gol, desviando de um obstáculo posicionado em frente às balizas e a $2 \mathrm{~m}$ da linha da grande área, finalizando com um chute ao gol com objetivo de acertar um alvo pré-determinado e fixado na área interna das traves. Dois arcos de 85 centímetros de diâmetro foram utilizados como alvos e colocados cada um em um ângulo do gol.

A partir dessa tarefa foi registrado o tempo total de sua execução, que foi compreendido do momento em que foi dado o comando de iniciar a tarefa (apito) até o momento da finalização ao gol, e o escore de acertos no alvo posicionado dentro do gol. Cada tarefa foi executada 5 vezes por cada atleta, sendo filmada sempre a $5^{\text {a }}$ tentativa, para a composição da sequência de vídeos a ser utilizado no protocolo de ativação do sistema de neurônios espelho.

Outro instrumento da pesquisa foi a sequência de vídeos que mostrou as atletas participantes do estudo realizando a atividade proposta e anteriormente citada. $\mathrm{O}$ vídeo foi exibido para o Grupo Neurônio Espelho (NE) e tinham como objetivo a estimulação do sistema de neurônios espelhos daquelas que o assistiram. As imagens eram compostas por diferentes atletas executando a tarefa que foi registrada no vídeo, e, de forma sincronizada com as imagens, era reproduzido o áudio com os comandos para o treinamento mental.

Por fim, foram utilizadas duas salas amplas na própria instituição que reuniram todas as condições necessárias para a realização dos testes, como controle de temperatura, de ruídos e de fontes de interferências nos instrumentos utilizados. 


\subsection{Procedimentos}

Todos os procedimentos para obtenção dos dados a partir dos instrumentos descritos foram feitos no Centro de Educação Física Almirante Adalberto Nunes (CEFAN), localizado na cidade do Rio de Janeiro - Brasil. Com a devida autorização dos responsáveis pelo Centro, foi realizada uma reunião com toda a comissão técnica para a explicação dos procedimentos e objetivos do trabalho. Posteriormente, a partir do consentimento da comissão técnica, uma reunião com as atletas foi realizada para esclarecimento das dúvidas e obtenção do termo de consentimento Livre e Esclarecido. Desta forma, foram definidos os dias para coleta dos dados pré-intervenção.

Para a coleta do padrão básico da atividade cerebral previamente foi ativado o filtro digital do equipamento com frequência de corte inferior e superior em 0,5 e $70 \mathrm{~Hz}$, respectivamente. Após isso, as atletas foram conduzidas individualmente a uma sala mantida a uma temperatura constante de $23^{\circ}$, e luminosidade e nível de ruídos controlados. Sentadas em uma cadeira com encosto e acolchoada com material isolante, posicionada longe de paredes e de outros instrumentos eletrônicos que pudessem produzir interferências, os eletrodos foram preparados e posicionados para a coleta dos dados durante um período de 3 minutos, onde o primeiro minuto não foi considerado na análise por se considerar que nesse período as atletas poderiam não estar em um estado de relaxamento ideal. Por fim, os dois minutos finais do exame foram analisados. Caso ocorresse algum evento no traçado durante esse tempo o mesmo era marcado e desconsiderado para determinação do padrão básico da atividade cerebral.

Determinado o padrão básico da atividade cerebral, foi avaliada a tomada de decisão através do software de avaliação do tempo de reação motora. Para tanto, primeiramente foi feita uma explicação de todo o procedimento do teste e permitidas 2 tentativas para que houvesse familiarização com o mesmo. Não havendo mais dúvidas por parte da atleta sobre o procedimento do teste, era solicitado que a mesma realizasse mais 3 registros da velocidade de reação, onde optou-se por ser considerado válido o menor tempo registrado para se ter um escore do melhor desempenho alcançado no teste. Vale ressaltar que o software onde foram realizados os testes foi configurado para estímulos mais complexos que envolviam tomar a decisão de apertar uma tecla específica do computador diante de 4 possíveis, no menor espaço de tempo, ou seja, era informado a atleta que entre 4 teclas pré-determinadas 1 seria apresentada aleatoriamente na tela, sendo necessário o pressionamento da tecla correspondente à imagem no menor tempo possível.

Posteriormente, a atleta foi encaminhada para o campo de treinamento onde realizou a tarefa para avaliação da habilidade motora. Individualmente, cada atleta executou cinco vezes a tarefa, de modo a ser filmada apenas na quinta repetição, por uma câmera apoiada em um tripé e posicionada na lateral do campo, a fim de capturar de forma ampla toda a área a ser utilizada pelas atletas, sem a necessidade de intervenção e deslocamento do equipamento durante a tarefa. A opção por se registrar apenas a última execução seguiu o mesmo critério adotado na avaliação da tomada de decisão, ou seja, utilizar para análise o melhor desempenho motor possível na tarefa. Sendo assim, por serem atletas, e por não se ter encontrado na literatura um número ótimo de tentativas necessárias para se passar do ciclo instabilidade-estabilidade, convencionou-se que a 5 tentativa seria a ideal para que tanto a ansiedade e nervosismo das primeiras tentativas quanto um eventual estresse físico/psicológico não comprometessem o desempenho técnico na tarefa.

Como os procedimentos pré-intervenção seguiram os mesmos critérios para todas as 
participantes, a randomização dos grupos foi feita através de sorteio antes do início do período de intervenção. Para isso, uma sequência numérica foi representada em papel de forma duplicada, de modo que um número em um dos papéis foi atribuído para cada atleta, que permaneceu com ela em mãos durante todo o sorteio, e o segundo papel com o número correspondente foi depositado em um recipiente. De modo alternado, o primeiro número sorteado foi designado para compor o grupo Neurônio Espelho (NE) (n=9), igualmente, o segundo número sorteado foi designado para compor o grupo Estimulação Cerebral (EC) $(n=8)$ e assim sucessivamente até ambos os grupos estarem formados.

No que diz respeito aos procedimentos de intervenção, em todos os dias de treino, as atletas do grupo NE receberam através do celular, um arquivo contendo a sequência de vídeos, mostrando as próprias atletas realizando a tarefa anteriormente gravada para este estudo. Juntamente as imagens do vídeo foi mixado um arquivo de áudio com o protocolo de treinamento mental, que tinha o objetivo de estimular nas atletas, não apenas a mentalização da sequência dos movimentos observados em vídeo, mas também a imaginação de si mesmas realizando esses movimentos. Os comandos eram dados em intervalos de 2 a 3 minutos de forma que a cada comando um aspecto específico do movimento era estimulado a mentalizado e/ou vivenciado mentalmente.

Para as atletas do grupo EC, foi enviado também através do celular, o mesmo protocolo de treinamento mental, porém associado à estimulação cerebral através das batidas binaurais, e sem as imagens de vídeo das atletas realizando a tarefa. Para isso, foi gravado um áudio em formato MP3 um protocolo de estimulação pré-definido para induzir estados de relaxamento concentrado favoráveis ao aprendizado. Concomitantemente ao som das batidas binaurais foram introduzidas às vozes de comando para o treinamento mental. Ambos os grupos, participaram dos protocolos pelo período de 15 minutos, diariamente antes dos treinamentos convencionais programados pela comissão técnica. Infelizmente devido a falta de financiamento para o estudo e limitações de acesso as atletas sempre antes dos treinamentos, não foi possível se fornecer equipamentos para que todas pudessem executar os arquivos enviados com a mesma qualidade de áudio e vídeo e nem se estar juntamente com as atletas sempre durante as sessões de estimulação.

Apesar das dificuldades de controle identificadas na intervenção poderem se configurar como elementos de viés, decidiu-se por manter os procedimentos de intervenção por se entender que tal situação pode ser comum a muitos clubes de futebol, e, justamente por esse motivo, conferir a presente pesquisa um caráter ainda mais aplicado a realidade especificamente do futebol feminino brasileiro. Após um período total de 7 semanas de intervenção foram repetidos todos os procedimentos e testes descritos na avaliação inicial, pré-intervenção.

\subsection{Análise Estatística}

Inicialmente a normalidade dos dados foi feita pelo teste de Shapiro Wilk. Determinada a característica paramétrica ou não paramétrica dos dados foram calculadas a tendência central, dispersão e extremos de cada conjunto de dados das variáveis em cada ensaio experimental feito na pesquisa.

As hipóteses foram testadas através de análises inferenciais dentro de cada grupo e entre os grupos, utilizando-se testes $\mathrm{T}$ pareados e não pareados dependendo da comparação feita. Para todas as análises foi adotado o índice de $95 \%$ de confiança para que os resultados representassem a realidade. 
Para as análises entre os grupos foi dado um tratamento diferente aos dados. Foram calculados e normalizados em $100 \%$, e a partir daí, determinado o percentual de aumento ou diminuição que cada variável registrou entre as avaliações. Esses escores dos grupos neurônios espelho e estimulação cerebral foram comparados pelos testes estatísticos apropriados. Todas as análises foram feitas através do programa GraphPad Prism 7 e serão apresentadas nas tabelas a seguir.

\section{RESULTADOS E DISCUSSÃO}

A primeira informação relevante para compreensão dos dados da pesquisa é sobre a normalidade. Tal informação é relevante para poder determinar o valor mais confiável para a tendência central, bem como os instrumentos estatísticos mais indicados para se testar as hipóteses. Sendo assim, verificou-se que no grupo que realizou o treinamento mental associado à ativação dos neurônios espelhos, todos os conjuntos de dados relacionados às variáveis tiveram um comportamento normal. A exceção foi o dado do tempo de execução da tarefa na avaliação pré-intervenção.

Tabela 1. Apresentação da frequência das ondas Alfa e SMR, desempenho cognitivo e motor dos participantes do grupo neurônios espelho antes e após a intervenção.

\begin{tabular}{|c|c|c|c|c|c|c|c|c|c|c|c|c|c|c|c|c|}
\hline \multicolumn{17}{|c|}{ Grupo Neurônios Espelho } \\
\hline & \multicolumn{12}{|c|}{ Eletroencefalograma (Frequência em mV) } & \multirow{3}{*}{\multicolumn{2}{|c|}{\begin{tabular}{|c|}
$\begin{array}{c}\text { Desemp. } \\
\text { Cognitivo }\end{array}$ \\
$\begin{array}{c}\mathrm{T} . \mathrm{de} \\
\text { Reação (s) }\end{array}$ \\
\end{tabular}}} & \multirow{3}{*}{\multicolumn{2}{|c|}{$\begin{array}{c}\begin{array}{c}\text { Desemp. } \\
\text { Motor }\end{array} \\
\text { T. de Exec. (s) }\end{array}$}} \\
\hline & \multicolumn{6}{|c|}{ Frequência Ondas Alfa } & \multicolumn{6}{|c|}{ Frequência Ondas SMR } & & & & \\
\hline & \multicolumn{2}{|c|}{$\mathrm{C} 3$} & \multicolumn{2}{|c|}{$\mathrm{C} 4$} & \multicolumn{2}{|c|}{$\mathrm{CZ}$} & \multicolumn{2}{|c|}{$\mathrm{C} 3$} & \multicolumn{2}{|c|}{$\mathrm{C} 4$} & \multicolumn{2}{|c|}{$\mathrm{CZ}$} & & & & \\
\hline & Pré & Pós & Pré & Pós & Pré & Pós & Pré & Pós & Pré & Pós & Pré & Pós & Pré & Pós & Pré & Pós \\
\hline Mín. & 7,3 & 7,2 & 7,0 & 7,4 & 7,3 & 7,6 & 7,0 & 6 & 6,6 & 5,9 & 6,8 & 6,2 & 0,37 & 0,38 & 7,2 & 6, \\
\hline Med & 11,6 & 11 & 10,9 & 11 & 11,7 & 11 & 8,8 & 8,7 & 8,7 & 8,2 & 8,9 & 8,8 & 0,47 & 0,53 & 7,9 & 7 , \\
\hline Máx & 19 & 18 & 19 & 17 & 21 & 17 & 13 & 15 & 13 & 14 & 14 & 14 & 0,88 & 1,1 & 10 & 8 \\
\hline M.A & 11,9 & 12 & 11,9 & 11 & 12,4 & 11 & 9,0 & 9,6 & 9,0 & 9,2 & 9,3 & 9,4 & 0,54 & 0,62 & 8,1 & 7,2 \\
\hline D.P. & 3,8 & 3,1 & 3,9 & 3,1 & 4,2 & 3,3 & 2,0 & 3 & 2,3 & 2,9 & 2,2 & 3,1 & 0,17 & 0,23 & 0,78 & 0,62 \\
\hline
\end{tabular}

* Destaque dado a tendência central de acordo com a normalidade dos dados. M.A.: média aritmética; Med.: Mediana; D.P.: Desvio Padrão.

Em relação ao eletroencefalograma, os números apresentados representam a frequência em milivolts das ondas cerebrais Alfa e SMR em cada ponto específico (C3, C4 e $\mathrm{CZ}$ ). Sobre a onda alfa, houve pequeno aumento da frequência média somente em relação ao ponto $\mathrm{C} 3$, de $11,9 \mathrm{mV}$ para $12 \mathrm{mV}$. Em relação aos pontos $\mathrm{C} 4$ e $\mathrm{CZ}$, observou-se uma diminuição da frequência média do momento pré para o pós-intervenção, de $11,9 \mathrm{mV}$ para 11 $\mathrm{mV}$ no ponto $\mathrm{C} 4$ e de $12,4 \mathrm{mV}$ para $11 \mathrm{mV}$ no ponto CZ. Sobre a onda SMR, houve um aumento da sua frequência média em todos os pontos, de $9,0 \mathrm{mV}$ para $9,6 \mathrm{mV}$ no ponto $\mathrm{C} 3$, de 9,0 $\mathrm{mV}$ para $9,2 \mathrm{mV}$ no ponto $\mathrm{C} 4$ e de $9,3 \mathrm{mV}$ para $9,4 \mathrm{mV}$ no ponto $\mathrm{CZ}$.

No que diz respeito à avaliação da tomada de decisão, os números representam em segundos a velocidade de reação motora, onde houve pequeno aumento do momento pré para o momento pós-intervenção, de 0,54 segundos para 0,62 segundos. Já, sobre a avaliação do desempenho motor, os números representam em segundos o tempo de execução da tarefa, 
havendo uma diminuição do momento pré para o momento pós-intervenção, de 7,9 segundos para 7,2 segundos.

Assim como feito com os dados do grupo neurônios espelho, os dados do grupo estimulação cerebral também passaram pelo mesmo tratamento antes de serem apresentados na tabela 2. Sendo assim, a análise da normalidade mostrou que para esse grupo todas as variáveis, nos momentos antes a após as intervenções, apresentaram comportamento normal.

Tabela 2. Apresentação dos dados do EEG, desempenho cognitivo e motor dos participantes do grupo estimulação cerebral antes e após a intervenção.

\begin{tabular}{|c|c|c|c|c|c|c|c|c|c|c|c|c|c|c|c|c|}
\hline \multicolumn{17}{|c|}{ Grupo Estimulação Cerebral } \\
\hline & \multicolumn{12}{|c|}{ Eletroencefalograma (Frequência em mV) } & \multirow{2}{*}{\multicolumn{2}{|c|}{$\begin{array}{c}\text { Desemp. } \\
\text { Cognitivo }\end{array}$}} & \multirow{2}{*}{\multicolumn{2}{|c|}{$\begin{array}{c}\text { Desemp. } \\
\text { Motor }\end{array}$}} \\
\hline & \multicolumn{6}{|c|}{ Frequência Ondas Alfa } & \multicolumn{6}{|c|}{ Frequência Ondas SMR } & & & & \\
\hline & \multicolumn{2}{|c|}{ C3 } & \multicolumn{2}{|c|}{$\mathrm{C} 4$} & \multicolumn{2}{|c|}{$\mathrm{CZ}$} & \multicolumn{2}{|c|}{ C3 } & \multicolumn{2}{|c|}{ C4 } & \multicolumn{2}{|c|}{$\mathrm{CZ}$} & \multicolumn{2}{|c|}{$\begin{array}{c}\text { T. de } \\
\text { Reação (s) }\end{array}$} & \multicolumn{2}{|c|}{ T. de Exec. (s) } \\
\hline & Pré & Pós & Pré & Pós & Pré & Pós & Pré & Pós & Pré & Pós & Pré & Pós & Pré & Pós & Pré & Pós \\
\hline Mín. & 6,2 & 6,4 & 5,9 & 6,3 & 6,2 & 6,5 & 5,7 & 6,3 & 6,2 & 6,6 & 6,0 & 6,1 & 0,3 & 0,44 & 6,6 & 6,4 \\
\hline Med & 12,8 & 15,1 & 13,1 & 15,3 & 12,2 & 14,1 & 8,7 & 9,9 & 8.5 & 9,7 & 7,9 & 9,6 & 0,6 & 0,62 & 8, & 7,4 \\
\hline Máx & 17,6 & 21,4 & 19,1 & 17,5 & 18,4 & 19,0 & 11 & 14 & 12 & 15 & 12 & 14 & 0,86 & 0,78 & 8,7 & 9,2 \\
\hline M.A & 12,2 & 14,4 & 12,4 & 14,0 & 11,8 & 13,6 & 8,4 & 10,1 & 8,9 & 10,5 & 8,1 & 9,2 & 0,59 & 0,61 & 7,9 & 7,5 \\
\hline D.P. & 4,6 & 4,7 & 4,6 & 4,1 & 4,4 & 4,4 & 1,8 & 2,8 & 1,8 & 3,1 & 1,7 & 2,4 & 0,2 & 0,12 & 0,67 & 0,85 \\
\hline
\end{tabular}

* Destaque dado à tendência central de acordo com a normalidade dos dados. M.A.: média aritmética; Med.: Mediana; D.P.: Desvio Padrão.

A tabela 2 mostra os dados descritivos do grupo estimulação cerebral nos momentos pré e pós-intervenção. Mostra também a tendência central, extremos e dispersão de cada um dos dados.

Em relação ao eletroencefalograma, observando a onda alfa, houve um aumento da frequência média nos 3 pontos do momento pré para o momento pós intervenção. De $12,2 \mathrm{mV}$ para $14,4 \mathrm{mV}$ no ponto $\mathrm{C} 3$, de $12,4 \mathrm{mV}$ para 14,0 no ponto $\mathrm{C} 4$ e de 11,8 para 13,6 no ponto CZ. Já sob observação da onda SMR, também houve aumento da frequência do momento pré para o momento pós-intervenção, de $8,4 \mathrm{mV}$ para $10,1 \mathrm{mV}$ no ponto $\mathrm{C} 3$, de $8,9 \mathrm{mV}$ para 10,5 $\mathrm{mV}$ no ponto $\mathrm{C} 4$ e de 8,1 para 9,2 no ponto $\mathrm{CZ}$.

No que diz respeito à avaliação do desempenho cognitivo através do teste de tempo de reação, também houve neste grupo um pequeno aumento do momento pré para o momento pós-intervenção, de 0,59 segundos para 0,61 segundos. Diferentemente, na avaliação do desempenho motor através do tempo de execução da tarefa, houve uma pequena diminuição do momento pré para o momento pós-intervenção, de 7,9 segundos para 7,5 segundos.

A fim de buscar compreender os efeitos sobre o desempenho esportivo da associação do treinamento mental com ambas as estratégias, a ativação dos neurônios espelho em um grupo e a estimulação cerebral não invasiva noutro grupo, faz se necessário não apenas analisar os dados coletados, mas também compará-los. 
Grupo Neurônios Espelhos

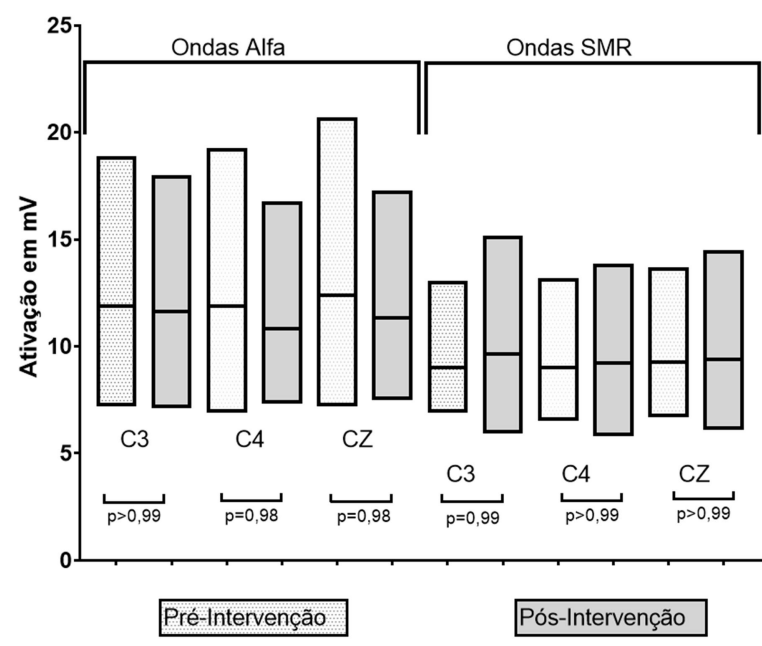

Grupo Estimulação Cerebral

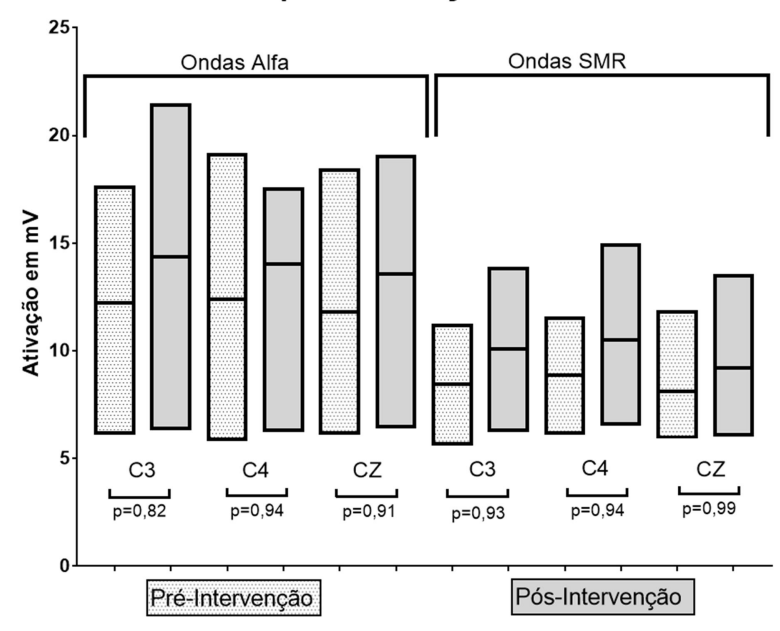

Figura 1. Comparação entre a atividades das ondas cerebrais Alfa e SMR nos momentos pré e pós-intervenção nos grupos que fizeram o treinamento mental associado a ativação dos neurônios espelhos e estimulação cerebral.

De acordo com a figura 1, ao se observar primeiramente o comportamento das ondas alfa no grupo NE, nota-se claramente uma pequena redução da sua atividade elétrica acompanhada de uma redução também na variabilidade do recrutamento de neurônios, o que significa dizer que do momento pré para o momento pós-intervenção, a atividade neural se mostrou mais homogênea, variando menos, possibilitando dizer que o comportamento das ondas alfa mostrou-se mais organizado, de modo a necessitar de uma menor ativação. De acordo com Luft e Andrade (2006), a onda alfa está associada com uma maior sincronia e menor esforço neural, consequentemente, relacionada com um indício de melhor aprendizado e possivelmente, melhor desempenho motor, uma vez que a atividade alfa relaciona-se com o desenvolvimento de estratégias cognitivas eficientes.

Por outro lado, ao se observar o comportamento das ondas SMR ainda no grupo NE, nota-se um pequeno aumento tanto em sua atividade média, quanto em sua variabilidade, diminuindo a homogeneidade do ritmo sensório motor, mas aumentando a atividade média da onda cerebral do momento pré para o momento pós-intervenção. De modo que se há maior atividade média, pode-se dizer que uma quantidade maior de neurônio pode estar sendo recrutada. Neste caso, o aumento da atividade média pode estar relacionado com o treinamento mental realizado por este grupo, onde de acordo com a Teoria Psiconeuromuscular (PNM), existe uma relação neurofisiológica direta entre uma experiência artificial imaginada e o desempenho real do indivíduo (FLEURY, 1998 apud. SCALON e SOUZA, 2004). Em outras palavras, no TM através da imagética pequenas quantidades de atividade neural podem ser medidas, de modo a criar marcas neurológicas atuantes sobre o indivíduo/atleta que realiza a experiência.

Já em relação ao grupo EC, ao se observar primeiramente o comportamento das ondas alfa nota-se claramente um aumento da atividade elétrica acompanhada de um pequeno aumento também na variabilidade do recrutamento de neurônios. Este comportamento se repetiu também em relação às ondas SMR. Acredita-se que neste caso, o aumento da atividade média de ambas as ondas, pode estar relacionado com a estimulação cerebral não invasiva via batidas binaurais aplicadas a este grupo. Segundo Brady e Stevens (2000) apud Arêas Neto (2010), a potencialização cerebral com batidas binaurais induz à estruturação e equalização da atividade cerebral, de modo a favorecer possivelmente, a manutenção da 
atenção e do foco na realização de tarefas. Para Guaranys, Santos e Silva (2010), a estimulação auditiva pode além de estimular a neuroplasticidade, aumentar o fluxo sanguíneo cerebral, aumentar a produção de determinados neurotransmissores e até mesmo equilibrar a atividade cortical entre os hemisférios cerebrais.
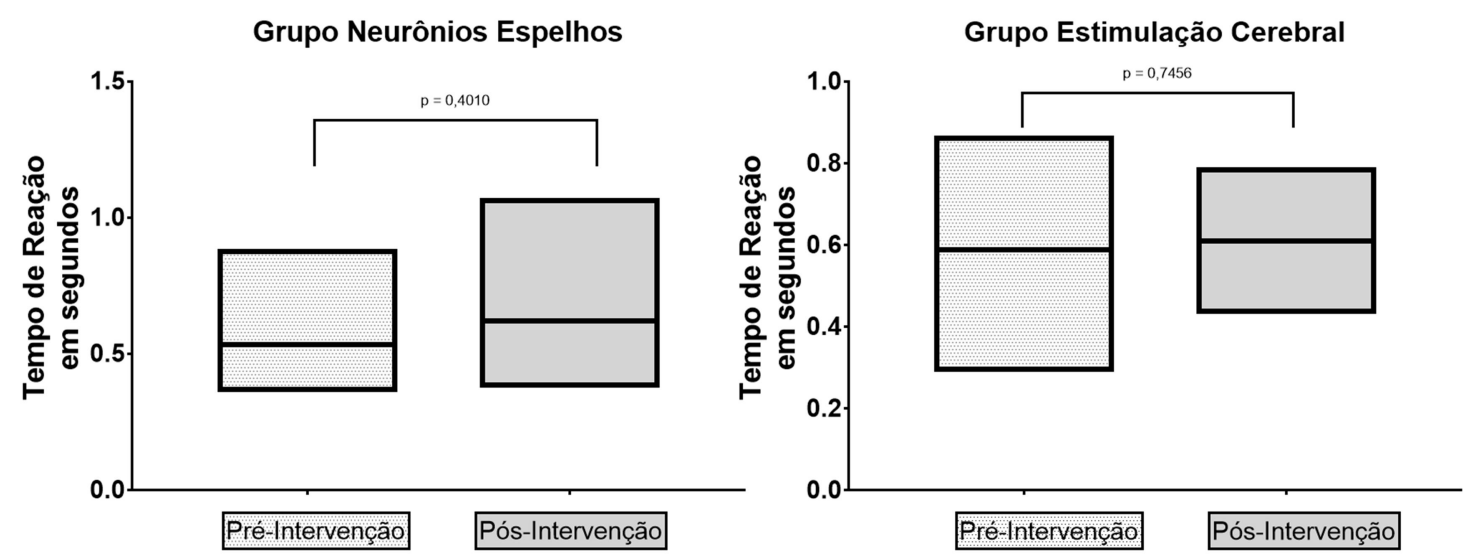

Figura 2. Comparação intra-grupos dos dados do tempo de reação motora nos momentos pré e pós-intervenção nos grupos que fizeram o treinamento mental associado à ativação dos neurônios espelhos e estimulação cerebral.

De acordo com a figura 2, ao se observar o tempo de reação motora em ambos os grupos, houve apenas uma pequena variação do momento pré para o momento pósintervenção, possivelmente pela composição da amostra, formada por atletas profissionais que de maneira constante tem a velocidade de processamento mental estimulada tanto nos treinos quanto pelas próprias exigências do desporto. Os jogos esportivos coletivos expressam um sistema de ações complexas exigindo do participante nas áreas física, técnica, tática, cognitiva e motora. Especialmente no futebol, fatores como variabilidade de sistemas ofensivo e defensivo, velocidade de tomada de decisão, imprevisibilidade do contexto ambiental e variações táticas, fazem parte do contexto do jogo (GIACOMINI et al, 2011), exigindo uma elevada capacidade de resposta e adaptação imediata por parte dos atletas e equipes dentro do contexto de cada treino ou partida durante uma competição (COSTA et al, 2009).
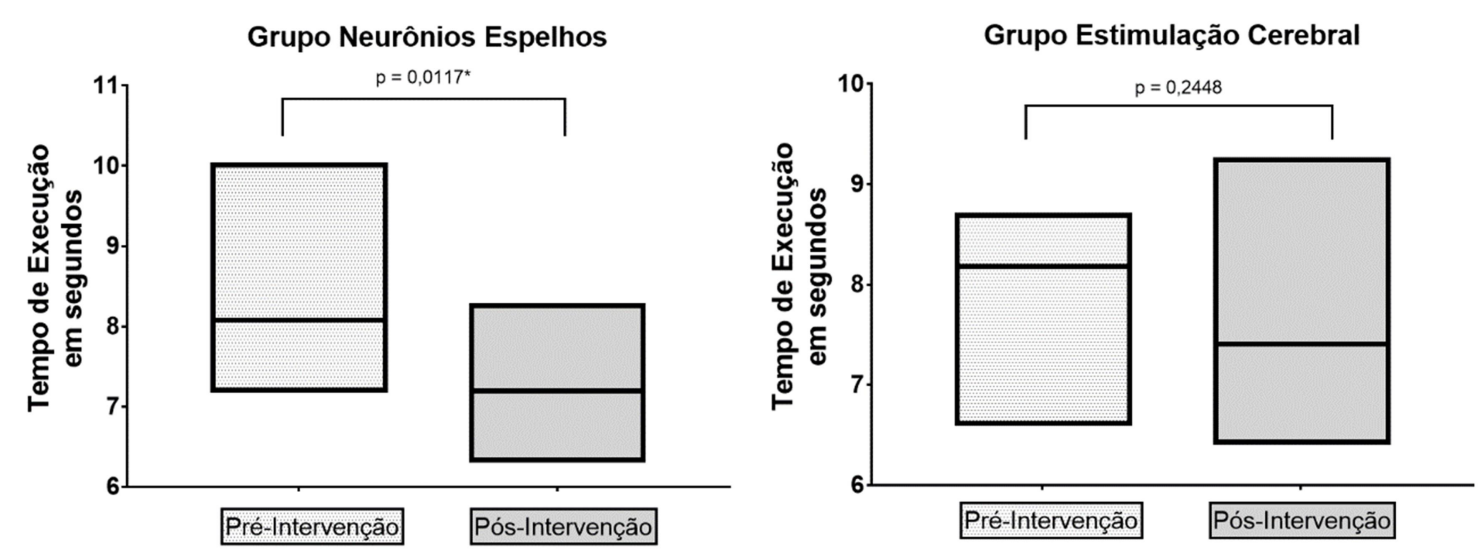

Figura 3. Comparação intra-grupos dos dados do tempo de execução da tarefa motora nos momentos pré e pós-intervenção nos grupos que fizeram o treinamento mental associado a ativação dos neurônios espelhos e estimulação cerebral. ${ }^{* *} \mathrm{p}<0,05$

A figura 3 mostra de forma clara, a diminuição do tempo de execução da tarefa em

Persp. online: biol. \& saúde, Campos dos Goytacazes, 34 (10) 16-32, 2020

https://ojs3.perspectivasonline.com.br 
ambos os grupos, do momento pré para o momento pós-intervenção, de modo a estar possivelmente relacionado com o treinamento mental praticado pelos dois grupos, uma vez que para Azevedo et al (2010), o referido ensaio mental é a possibilidade de atalho para o aprendizado motor, sendo o TM uma prática colaboradora, em relação ao tempo, para formulação de um novo engrama motor objetivando uma recente ou complexa atividade motriz a ser realizada. De acordo com Battaglia et. al. (2014) apud. Fortes (2017), a imaginação acede ao córtex motor e gera ativação neuromuscular similar a realização de uma tarefa motora. Tal pensamento corrobora com o pensamento de Franco (2000) apud Souza (2004), de que um movimento imaginado e exercitado mentalmente é capaz de produzir as chamadas microcontrações, que resulta em uma melhora da coordenação neuromuscular, devido a uma maior irrigação de sangue que é constatada na musculatura envolvida. Desta forma, é através do treinamento mental (imagética motora) que diversas áreas do sistema nervoso central, responsáveis pelo planejamento e execução do movimento podem ser ativadas, configurando a possibilidade de redução do tempo e possível aumento do desempenho motor (LOUIS et. al. 2008 apud. AZEVEDO et al, 2010).

Neste contexto, para os participantes do grupo neurônios espelho, o efeito provocado mostrou-se estatisticamente significativo, havendo neste grupo também, um aumento no percentual de acerto da tarefa motora. Este aumento foi de 4,4\% no momento pré-intervenção, para 7,5\% no momento pós-intervenção. Destaca-se a relevância de tal resultado, haja vista a complexidade da tarefa e a composição da amostra, formada por atletas profissionais com experiência na prática do desporto, de acordo com a sua idade e tempo de atuação no futebol profissional.

Enquanto ao grupo estimulação cerebral, em relação ao momento pós-intervenção, mesmo não havendo um aumento no percentual de acerto da tarefa motora, observou-se de forma clara que a maioria das atletas participantes do estudo executou toda a tarefa e finalizou, objetivando o alvo, de forma mais organizada e melhor, se comparado ao momento pré-intervenção. Ou seja, mesmo não havendo acertos efetivos, as finalizações foram visivelmente melhores, chegando bem próximas ao alvo estabelecido, que neste caso, se tratou de dois arcos de apenas 85 centímetros de diâmetro.

Tabela 3. Apresentação dos valores de p referente às comparações entre os grupos, dos dados normalizados das ondas cerebrais, tempo de reação motora e velocidade de execução da tarefa.

\begin{tabular}{|c|c|c|c|}
\hline & Análise de Variância & $\begin{array}{l}\text { Teste T Não } \\
\text { Pareado }\end{array}$ & $\begin{array}{l}\text { Teste T Não } \\
\text { Pareado }\end{array}$ \\
\hline & $\begin{array}{c}\text { Atividade } \\
\text { Eletroencefalográfica } \\
\text { das ondas Alfa e SMR } \\
\end{array}$ & $\begin{array}{c}\text { Tempo de Reação } \\
\text { Motora }\end{array}$ & $\begin{array}{l}\text { Velocidade de } \\
\text { Execução da } \\
\text { Tarefa Motora } \\
\end{array}$ \\
\hline & $\begin{array}{c}\text { Grupo Neurônios } \\
\text { Espelho } \\
\text { X } \\
\text { Grupo Estimulação } \\
\text { Cerebral }\end{array}$ & $\begin{array}{c}\text { Grupo Neurônios } \\
\text { Espelho } \\
\text { X } \\
\text { Grupo Estimulação } \\
\text { Cerebral }\end{array}$ & $\begin{array}{c}\text { Grupo Neurônios } \\
\text { Espelho } \\
\text { X } \\
\text { Grupo } \\
\text { Estimulação } \\
\text { Cerebral }\end{array}$ \\
\hline $\mathrm{P}$ valor & 0,9592 & 0,9926 & 0,3123 \\
\hline
\end{tabular}


De acordo com a tabela 3, não houve diferença estatisticamente significativa entre os grupos, no que diz respeito às comparações dos resultados alcançados por cada um deles, nas variáveis observadas na pesquisa. Em outras palavras, significa dizer que ambas as intervenções provocaram efeitos semelhantes em ambos os grupos.

Outros estudos também têm demonstrado resultados positivos das estratégias utilizadas nesta pesquisa. Sobre a eficácia do treinamento mental, Fortes et. al. (2017) concluíram que o treinamento foi eficiente para maximizar o desempenho no arremesso de lance livre de jovens atletas de basquetebol, corroborando com Azevedo et al (2010) que verificou a influência da imagética motora na performance esportiva de pré-adolescentes praticantes de futsal.

Sobre a estimulação cerebral via estímulos auditivos, um estudo feito por Calomeni et. al. (2008) demonstrou a eficiência do estímulo por batidas binaurais no auxílio da aprendizagem, ao ter como resultado uma melhora na atenção concentrada e na memória de trabalho da criança hiperativa. Dado sustentado por Lima (2014), que verificou que a estimulação cortical, por via auditiva, realizada por meio de batidas binaurais, mostrou-se valiosa na melhora dos níveis de atenção de crianças com TDAH. Por último, no que diz respeito à ativação do sistema de neurônios espelho, Santos-Naves et al (2014) investigou o efeito da demonstração na aprendizagem do saque japonês do voleibol, verificando melhoras no escore e no padrão de movimento dos indivíduos participantes do estudo.

\section{CONCLUSÕES}

A análise dos resultados do estudo não permite conclusões definitivas sobre os efeitos das intervenções nas atletas e condições testadas. Cabe ressaltar que o que se buscou foi verificar qual combinação (treinamento mental + Estimulação cerebral não-invasiva, ou, treinamento mental + ativação dos neurônios espelho) poderiam ser mais eficazes na melhoria do desempenho de uma tarefa motora no futebol. Nesse sentido, mesmo com uma amostra limitada as atletas do time com condições físicas de realizar a tarefa proposta, e pelas mesmas estarem em plena temporada de jogos, os dados mostram algumas diferenças interessantes entre o desempenho pré e pós-intervenção de cada grupo que apontam para uma provável influencia no desempenho da técnica esportiva específica.

Destaca-se certa limitação do estudo no que diz respeito ao controle de modo mais exigente sobre a realização das intervenções feitas pelas atletas participantes do estudo. Além de atletas de futebol, as participantes do estudo são praças da marinha do Brasil que fícam alojadas no próprio CEFAN onde treinam. Dessa forma, a divisão entre o tempo para os treinamentos e para as tarefas inerentes as rotinas militares impedia que os responsáveis pelas intervenções estivessem sempre presentes no momento das mesmas. Assim, ficou a cargo do treinador garantir que as atletas que realizassem os protocolos de intervenção específicos em seus alojamentos sempre 15 minutos antes do início das sessões de treinamento com o máximo de atenção possível.

Apesar do grande interesse das atletas em melhorar suas performances e a garantia do treinador que todas eram estimuladas e cobradas diariamente para realização das intervenções, não é possível garantir nesse estudo que as condições para a realização das intervenções foram sempre ótimas para todas as atletas. Além disso, outra interveniência que pode ter influenciado nos resultados vem do fato que a única maneira viável dentro do contexto apresentado para estimulação dos neurônios espelho foi através da utilização do celular para 
assistir os vídeos. Fator que pode ter causado certa dificuldade na percepção das atletas em relação aos detalhes das técnicas que deveriam ser treinadas mentalmente.

Todavia, era de se esperar que diante das dificuldades identificadas os resultados de todas as análises tenderiam para a hipótese nula. Porém, foi possível notar que ambas as intervenções promoveram redução no tempo de execução da tarefa, sendo essa diminuição estatisticamente significante no grupo NE, o que pode ser extrapolado para um melhor desempenho técnico da tarefa. Além disso, todo procedimento experimental aqui utilizado foi adaptado para a realidade que impera na maioria dos clubes de futebol feminino brasileiros mostrando que, mesmo sem resultados estatisticamente significativos, tem boa aplicação no campo prático. Mesmo assim, sugere-se que novas pesquisas sejam realizadas se utilizando das mesmas estratégias em melhores condições e com um maior controle sobre a realização da intervenção, a fim de verificar se melhores condições na aplicação do treinamento mental associado a outras técnicas poderão influenciar nos resultados do estudo.

\section{REFERÊNCIAS}

ARÊAS NETO, N.T.; MORALES, A.P.; CALOMENI, M.R.; VIANA, M.A.S.; \& SILVA, V.F. Estimulação cortical: efeitos agudos sobre variáveis bioperacionais em jogadores armadores de basquetebol. Revdig, efdeportes. com, Buenos Aires, v. 15, p. 150, 2010. Disponível em: <http://www.efdeportes.com/efd150/estimulacao-cortical-em-jogadores-debasquetebol.htm>.

AZEVEDO, P.A.; NUNES, R.A.; JAKUBOVIC, B.K.M.; MOURÃO, J.G.; \& SILVA, A.L.S. A influência da imagética motora na performance esportiva de atletas de futsal. RevDigBuenos Aires, v. 14, n. 140, p. 77-85, 2010.

BISCAIA, P.; COELHO, E.; MENDO, A. H.; \& ALVES, J. Processamento da informação e antecipação em jogadoras de andebol de elite: Da formação ao alto nível: da formação ao alto nível. Revista iberoamericana de psicología del ejercicio y el deporte, v. 13, n. 2, p. 179191, 2018.

CALOMENI, M.R.; ROCHA, J.A.M.D.S.; SILVA, A.P.R.D.; RIBEIRO, L.H.B.; MARQUES, L.; SIZA, M.A.F.; \& SILVA, V.F.D. Brain stimulation used as biofeedback training for recovery of motor functions deteriorated by stroke. Arquivos de neuropsiquiatria, v. 71, n. 3, p. 159-164, 2013.

CALOMENI, M.R.; DA SILVA, V.F.; VELASQUES, B.B.; FEIJÓ, O.G.; \& BITTENCOURT, J.M. Modulatory effect of association of brain stimulation by light and binaural beats in specific brain waves. Clinical practice and epidemiology in mental health: $\mathbf{C P}$ \& $\mathbf{E M H}, \quad$ v. 13, p. 134, 2017. Disponível em: $<$ https://www.ncbi.nlm.nih.gov/pmc/articles/PMC5712641/>. Acesso em: Março de 2019.

CALOMENI, M.R. PELLICIARI, B.C.; NETO, N.T.A.; DANTAS, E.H.M.; \& DA SILVA, V.F. Treinamento Mental e Estimulação Cerebral: Efeitos $\mathrm{Na}$ Memória de trabalho de Crianças com Dificuldade de Aprendizagem. Humanas Sociais \& Aplicadas, v. 8, n. 22, 2018.

Disponível em: $<$ http://www.seer.perspectivasonline.com.br/index.php/humanas_sociais_e_aplicadas/artic le/view/1214/1043>. Acesso em: Março de 2019.

CALOMENI MR et. al. Estimulação fótica e auditiva: Efeitos em criança hiperativa. 
Perspectiva online, v.5, n.2, p. 34-42, 2008.

CONDE, E.Q.; FILGUEIRAS, A.; MATOS, J.B.; DE ANDRADE, R.B.; RIBEIRO, P.S.; DE LACERDA MIRANDA, A.; \& TAKASE, E. Aspectos neurais em chutes no futebol: um estudo de caso sobre a adaptação de técnicas cinemétricas para avaliação e treinamento cognitivo. Ciências \& Cognição, v. 17, n. 2, 2012.

COSTA, I.T. DA SILVA, J.M.G.; GRECO, P.J.; \& MESQUITA, I. Princípios Táticos do Jogo de Futebol: conceitos e aplicação. Motriz, Rio Claro, v.15 n.3 p.657-668, jul./set. 2009.

FILGUEIRAS, A. SANTANA, C.M.; CONDE, E.F.; \& RIBEIRO, P. O uso da imagética mental como técnica para melhora do aproveitamento no futebol de campo: um estudo de caso. Revista Coleção Pesquisa em Educação Física, v. 11, n. 4, p. 167-174, 2012.

MATIAS, C.J.A.S.; \& GRECO, P.J. Cognição \& ação nos jogos esportivos coletivos. Ciências \& Cognição, v. 15, n. 1, p. pp. 252-271, 2010.

DARONCH, C.; DE SOUZA PETERSEN, R.D.; SPINDOLA, M.M.; VAZ, M.A.; DE OLIVEIRA, L.F.; \& GEREMIA, J.M. Bailarinas $\mathrm{x}$ voleibolistas: efeito de diferentes treinamentos motores sobre o sinal eletroencefalográfico. Revista Brasileira de Ciências do Esporte, v. 38, n. 4, p. 384-391, 2016.

LIMA, A.P. CARDOSO, F.B.; LIMA, I.D.C.; FRAGEL-MADEIRA, L.; \& SHOLLFRANCO, A. Avaliação da eficácia de um programa de estimulação cortical para melhora da atenção de crianças com TDAH. Saúde (Santa Maria), v. 40, n. 1, p. 71-76, 2014.

LIMA, A.P.; CARDOSO, F.B.. A eficácia da estimulação cortical no desenvolvimento perceptivo motor de adolescentes com síndrome de Down. ConScientiae Saúde, v. 16, n. 3, p. 311-317, 2017.

FERNANDES, C.N. et al. Técnica espelho: para compreender este fenômeno num grupo comunitário de ginástica para a terceira idade. 2010. Disponível em: https://repositorio.ufsc.br/handle/123456789/191429

FORTES, L.S. CARMO, Y.A.F.D.; CRUZ, R.B.D.S.F.; LIMA, E.T.P.D.; \& MANSUR, H.N. Efeito do treino mental no desempenho do arremesso de lance livre em jovens basquetebolistas. Motricidade, v. 13, n. 3, p. 04-12, 2017. Disponível em: $<$ http://www.scielo.mec.pt/scielo.php?script=sci_arttext\&pid=S1646-107X2017000400002>. Acesso em: Março de 2019.

GIACOMINI, D.S.; SOARES, V. O.; SANTOS, H.F., MATIAS, C.J.; \& GRECO, P. J; o conhecimento tático declarativo e processual em jogadores de futebol de diferentes escalões. Motricidade, v. 7, n. 1, p. 43-53, 2011.

GONÇALVES, F. Treino da Velocidade dos Jovens no Voleibol-Exercícios para o Desenvolvimento do Tempo de Reação Complexo e da Velocidade de Execução. 2014. Disponível em: http://hdl.handle.net/10400.24/148

GUARANYS, C.E.L.B.; SANTOS, J.B; SILVA, V.F. Estimulo Auditivo Na Melhora Do Foco De Atenção. FiepBulletin. Volume 80. SpecialEdition. ARTICLE II - 2010.

LAMEIRA, A.P.; GAWRYSZEWSKI, L.G.; PEREIRA JR, A. Neurônios espelho. Psicologia 
UsP, v. 17, n. 4, p. 123-133, 2006.

OLIVEIRA, S. O efeito do treinamento da imaginação, na melhora do gesto 6."flic com as mãos" na ginástica rítmica feminina, em atletas de 8 a 12 anos de idade [dissertação]. Curitiba: Universidade do Paraná, 2007. Disponível em: $<$ https://www.acervodigital.ufpr.br/bitstream/handle/1884/11734/VERSAO?sequence $=1>$.

Acesso em: Março de 2019.

OKAZAKI, V. H. A. Reaction Time (version 1.0)[Adapted Software]. Obtido de http://okazaki. webs. com, 2011.

PEREIRA, N.F.; SANTOS, R.G.M.; CILLO, E.N.P. Arbitragem no futebol de campo: estresse como produto de controle coercitivo. Revista brasileira de psicologia do esporte, $v$. 1, n. 1, 2018.

RIBEIRO JÚNIOR, S.M.S.; AZEVEDO, M.M.D.A.; JORGE, F.S.; MORALES, A.P.; \& SILVA, V.F.D. Efeito da estimulação cortical na variável tempo de reação e performance hábil-motriz-cognitiva em jovens atletas de futebol. Motriz rev. educ. fís.(Impr.), v. 16, n. 4, p. 869-879, 2010. Disponível em: <http://www.scielo.br/pdf/motriz/v16n4/a07v16n4>

RIPOLL, H. Do tratamento da informação à tomada de decisão no desporto. Manual de psicologia do desporto para treinadores, p. 295-314, 2011.

SANTOS-NAVES, S.P.; BENDA, R.N.; JUNQUEIRA, A.H.M.; ALVES, G.M.; VELLOSO, A.L.P.P.; \& UGRINOWITSCH, H. Efeito da demonstração distribuída na aprendizagem do saque do voleibol. Revista Brasileira de Educação Física e Esporte, v. 28, n. 4, p. 629-639, 2014.

SILVA, V.F.D.; CALOMENI, M.R.; NUNES, R.A.M.; PIMENTEL, C.E.; MARTINS, G.P.; OLIVEIRA, P.D.C.A.; \& SILVA, P.B. Brain stimulation used as biofeedback in neuronal activation of the temporal lobe area in autistic children. Arquivos de neuro-psiquiatria, v. 74, n. 8, p. 632-637, 2016.

SCALON, R.M.; SOUZA, A.P.S. O Treinamento Mental como uma variável significativa na performance de atletas e na aprendizagem de habilidades motoras. Lecturas: Educación física y deportes, n. 75, p. 16, 2004.

TENENBAUM, G. Expert athletes: An integrated approach to decision making. Expert performance in sports, p. 191-218, 2003.

THOMAS, J.R.; NELSON, J.K.; SILVERMAN, S.J. Métodos de pesquisa em atividade física. Artmed Editora, 2009. 